\title{
Novel Aspects of Wedge Filling by Liquid Helium
}

\author{
R. Mayol $^{a}$, F. Ancilotto ${ }^{b}$, M.Barranco ${ }^{a}$, \\ E. S. Hernández ${ }^{c}$ and M. Pi ${ }^{a}$ \\ ${ }^{a}$ Departament E.C.M., Facultat de Física, and $I N^{2} U B$ \\ Universitat de Barcelona, 08028 Barcelona, Spain \\ ${ }^{b}$ Dipartimento di Fisica 'G. Galilei', Università di Padova, I-35131, \\ and DEMOCRITOS National Simulation Center, Italy \\ ${ }^{c}$ Departamento de Física, Facultad de Ciencias Exactas y Naturales, \\ Universidad de Buenos Aires, 1428 Buenos Aires, \\ and Consejo Nacional de Investigaciones Científicas y Técnicas, Argentina
}

We discuss several features of the condensation of helium in linear wedges with $\mathrm{Cs}$ and $\mathrm{Na}$ walls, such as the existence of pre-filling jumps, and the relationship to prior calculations of the growth of $1 D$ helium stripes in the outer grooves of carbon nanotube bundles. We also present preliminary results of condensation between parallel Cs plates.

PACS numbers 67.60.-g, 67.70.+n,61.46.+w

\section{INTRODUCTION}

When an adsorbed film grows on a substrate, the geometry of the latter strongly influences the wetting behavior of the fluid. Presently, it is possible to carry out noninvasive experiments that resolve the imperfect interfacial structure of real surfaces, ${ }^{1,2}$ as well as to manufacture patterned surfaces in the micrometer ${ }^{3}$ and in the nanometer ${ }^{2}$ scales. Among the various defects and irregularities that may appear on a macroscopically planar surface, grooves and wedges are simple but fairly realistic structures allowing for theoretical studies, and classical theories for the condensation of fluids into wedges have been firmly established through the 90 's. ${ }^{4-7}$ Recent measurements ${ }^{8}$ of adsorption of Ar on micropatterned Si confirmed a theoretical prediction ${ }^{9}$ regarding the crossover between the planar and the geometrycontrolled regimes; moreover, it has been suggested that adsorption of liquids into wedges might permit to observe effects of pre-wetting. ${ }^{6}$

Early theoretical approaches ${ }^{4-7}$ aim at describing wedge filling and wedge 


\section{R. Mayol et al.}

wetting in the macro and mesoscopic scales and at finite temperatures. It is then of interest to investigate the extent to which general features persist in the nanoscale and at the lowest attainable temperatures for superfluid helium. Recently, ${ }^{10}$ we have presented the first complete calculation of the twodimensional atomic density $\rho(x, z)$ and energetics of liquid ${ }^{4} \mathrm{He}$ confined in a concave alkaline wedge, infinite in the $y$-direction, as a function of the opening angle $2 \varphi$ of the walls, within finite-range density functional theory (FRDFT). ${ }^{11}$ Starting from a pancake-shaped helium sample of nanoscopic extension in the $(x, z)$ plane, we have found a sequence of particle distributions and meniscii shapes that includes: a) one filling transition on the nonwettable $\mathrm{Cs}$ for an aperture $\varphi_{F}=\pi / 2-\theta$, with $\theta$ the contact angle of helium on $\mathrm{Cs}$; b) an angular range where the meniscus is concave; c) a threshold $\varphi_{E}$ for the appearance of metastable 'bridge' configurations (for Cs and Na wedges), where the wedge apex becomes void of helium. Such a drying/emptying transition is not expected on the basis of macroscopic thermodynamic arguments and is an important, nontrivial effect, in view of recent experimental evidence on the stability of micropuddles on rough Cs surfaces exposed to helium vapor. ${ }^{12}$

\section{STABILITY OF HELIUM SYSTEMS IN THE WEDGE}

Within FRDFT, the equilibrium helium density $\rho(x, z)$ has been obtained minimizing the zero temperature grandpotential per unit transverse lenght, whose variation with respect to $\rho$ gives rise to the integrodifferential Euler-Lagrange equation

$$
\left[-\frac{\hbar^{2}}{2 m} \nabla^{2}+V(\rho)+V_{s}(x, z)\right] \sqrt{\rho(x, z)}=\mu \sqrt{\rho(x, z)},
$$

where $\mu$ is the chemical potential, $V(\rho)$ is the first functional derivative of the potential energy density per unit length, and $V_{s}(x, z)$ is the confining field, chosen as the summation of two planar potentials, each of the form given in Ref. 13 , at an angle $2 \varphi$.

For Cs walls, between the filling angle $2 \varphi_{F}=110^{\circ}$ and the emptying one $2 \varphi_{E}=52^{\circ}$, the corresponding configurations for helium in the wedge are stable, while they are metastable above $\varphi_{F}$ and below $\varphi_{E} .{ }^{10}$ Condensation, associated to negative grandpotential, is accompanied by pre-filling jumps in the linear density at the threshold. The mechanism of condensation is better understood by examining the adsorption isotherms $\mu(n)$, where $n$ is the linear density, in a wedge with fixed aperture. For all angles in the stable domain, condensation takes place with a jump in the linear density 


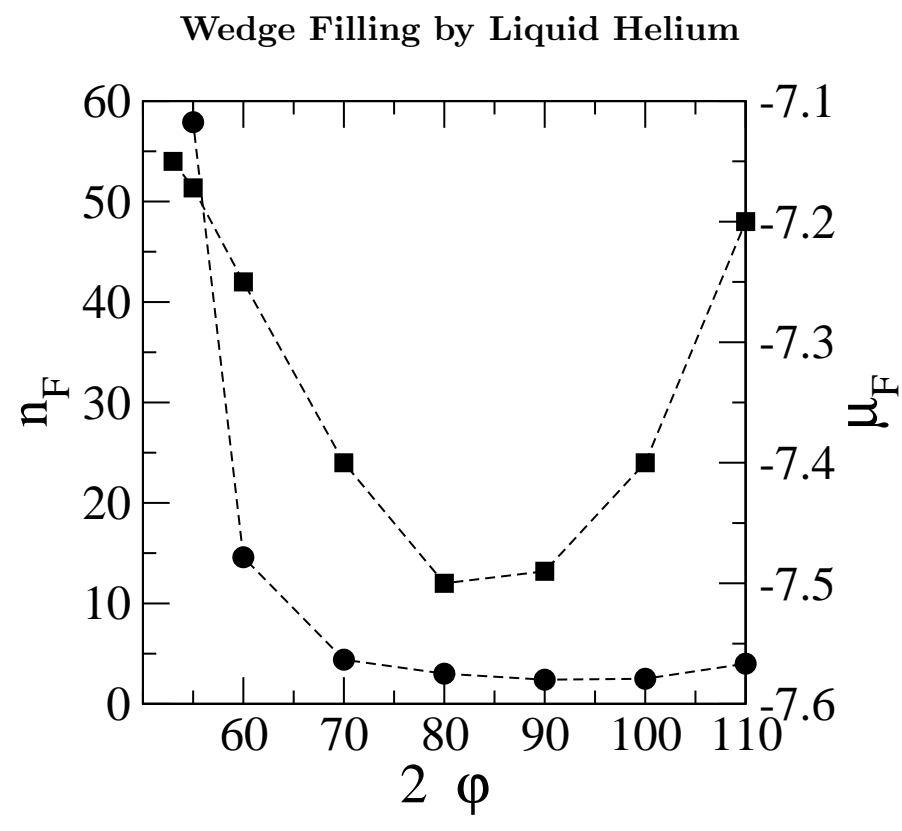

Fig. 1. Pre-filling jump $n_{F}$ (in $\AA^{-1}$, indicated with circles) and corresponding chemical potential $\mu_{F}$ (in $\mathrm{K}$, squares) obtained from the adsorption isotherms of helium on Cs wedges as a function of wedge opening (in degrees). The lines are drawn to guide the eye. The divergence at an angle slightly above $110^{\circ}$ is not shown.

from $n=0$ to a finite value $n_{F}$. The usual Maxwell construction permits one to identify the chemical potential and density at the corresponding jump for each aperture. This is indicated in Fig. 1, where we show the size of the pre-filling jump $n_{F}$ together with the corresponding chemical potential $\mu_{F}$, as functions of the angle.

For apertures above the filling and below the emptying ones the grandpotential per particle is positive, ${ }^{10}$ i.e., helium does not condense in such wedges. The linear density $n_{F}$ is finite within the condensation range and diverges at the filling and emptying transitions, going through a shallow minimum around $2.4 \AA^{-1}$ near $2 \varphi=100^{\circ}$. The chemical potential $\mu_{F}$ at the jump displays a minimum of about $-7.5 \mathrm{~K}$ at a smaller opening of about $80^{\circ}$. For the strong $\mathrm{Na}$ adsorber, in the angular interval here displayed, only the divergence at $\varphi_{E}$ shows up; starting from large angles, the jump density $n_{F}$ decreases monotonically to a minimum around $0.11 \AA^{-1}$. In either case, we have found that for the linear densities under consideration, the helium bridges that appear below $2 \varphi_{E}$ are metastable (i.e. have higher energies) with respect to helium pancakes deposited on planar surfaces. In the metastability region the pancake, which is a symmetry breaking configuration, is energetically favored with respect to the axially symmetric 


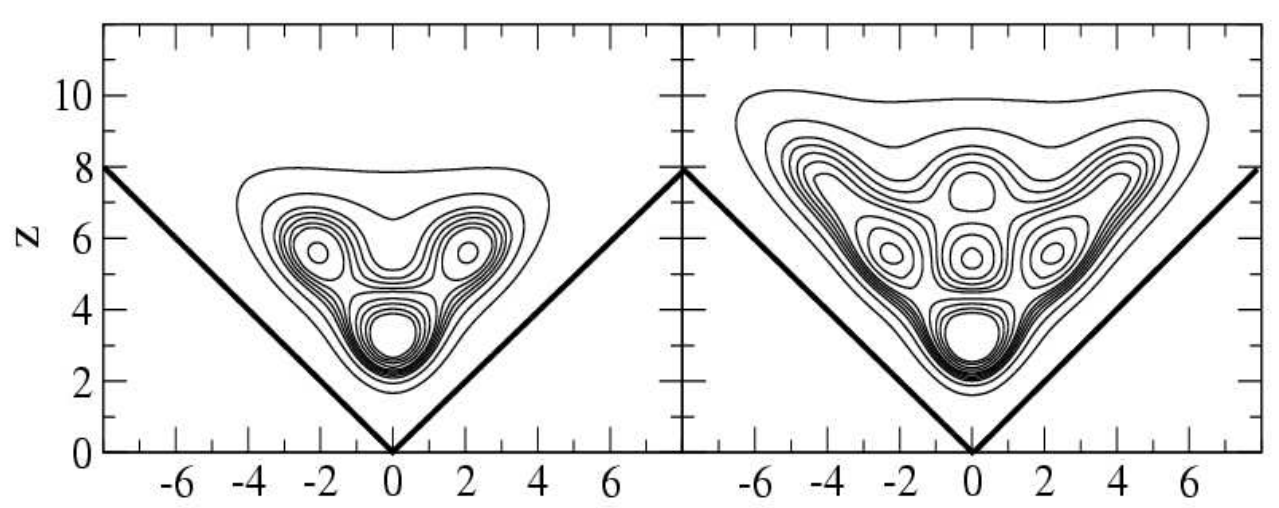

Fig. 2. Equidensity lines for helium in a $\mathrm{Na}$ wedge with opening of $90^{\circ}$, for linear densities: $n=0.5 \AA^{-1}$ (left panel) and $1 \AA^{-1}$ (right panel). Lengths are in $\AA$

configuration.

\section{THE LOW DENSITY REGIME}

In Ref. 14 it was shown by means of classical, grandcanonical Monte Carlo calculations, that a sequence of phases appears in ${ }^{4} \mathrm{He}$ adsorbed on the outer surface of carbon nanotube bundles. Ground state calculations for $\mathrm{Ne}$ and $\mathrm{CH}_{4}$ in a groove between neighbouring tubes give rise to a $1 \mathrm{D}$ phase ${ }^{14}$ located at the vertex -at the lowest vapor pressure- followed by 3-, 7- and 8-stripe phases consisting of symmetric arrangements of quasi-1D adsorbed samples, for increasing pressure. We have found that similar high density patterns are also present near the wedge apex. This is illustrated in Fig. 2 where quasi-1D structures are clearly visible close to the wedge apex (whose highest density peak is about three times the saturation density), followed by symmetric stripe configurations sitting in a dilute helium environment, for increasing amount of helium. Similar patterns, with smoother density oscillations, are present even for the weakly attractive Cs substrate.

These results suggest a scenario for the first stages of helium condensation in wedges, that can be traced to the layering process on planar substrates of different strengths. The latter can be summarized as follows. On flat alkali substrates, helium film growth close to the surface takes place through addition of successive liquid layers. Instead, on a strong adsorber such as graphite, the first two layers are $2 \mathrm{D}$ solids. Our results indicate that 
Wedge Filling by Liquid Helium

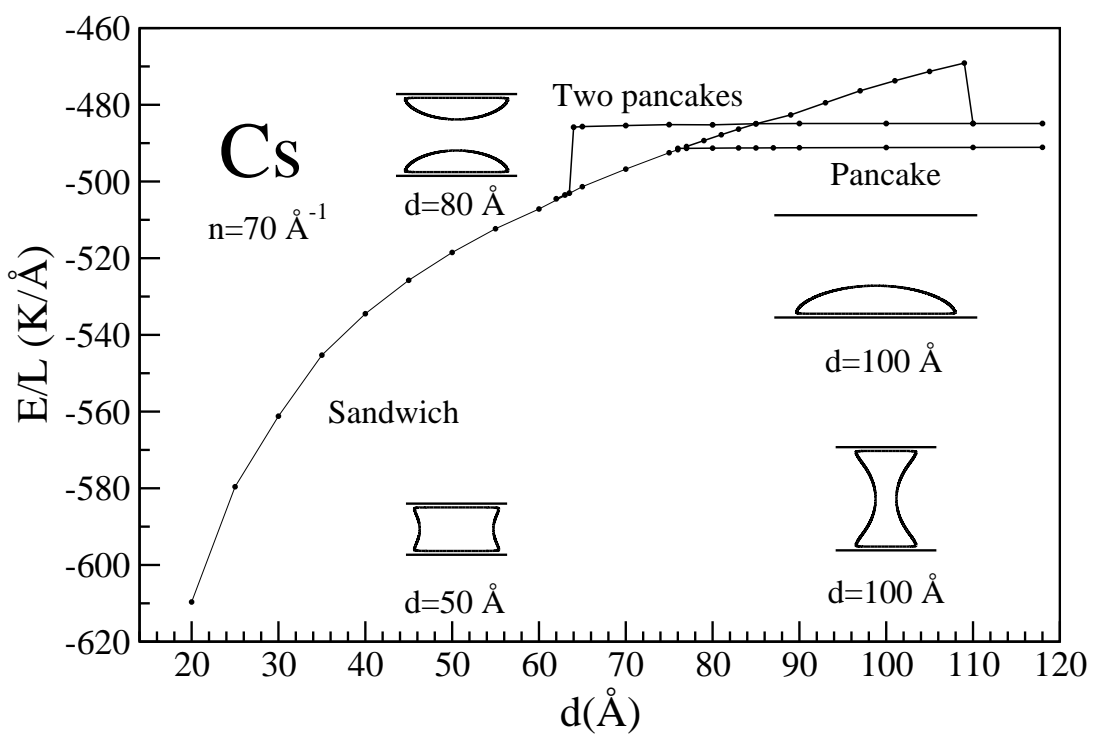

Fig. 3. Energy per unit length $E / L$ for a helium density $n=70 \AA^{-1}$ as a function of the distance $d$ between two parallel Cs plates. The lines are drawn to guide the eye. Several configurations obtained at the indicated $d$ values are displayed showing the equidensity line corresponding to half the liquid saturation density value.

the growth of ${ }^{4} \mathrm{He}$ in alkali wedges occurs through the addition of succesive quasi-1D stripes, that play the role of the former quasi-2D planar shells. In fact, one might regard these quasi-1D structures as precursors of the quasi$2 \mathrm{D}$ layers that will appear adjacent to the walls, far from the apex, as the amount of material increases. By contrast, in the strongly attractive grooves between carbon nanotubes, this growth takes place by means of 1D stripes, which for increasing vapor pressure, give rise to a $1 \mathrm{D}$ monolayer adjacent to the nanotube walls, as anticipated in Ref. 14.

\section{CONDENSATION BETWEEN PARALLEL WALLS}

Finally, we present preliminary results for the condensation between parallel Cs plates, which might represent the limiting situation, very far from the apex wedge, when the wedge opening approaches zero.

Fig. 3 displays the energy per unit length corresponding to a helium linear density $n=70 \AA^{-1}$, as a function of the distance $d$ between the walls. At distances below $75 \AA$, a sandwich-like configuration -equivalent to the wedge one in this geometry- extends symmetrically between the plates, 


\section{R. Mayol et al.}

and is the stable one. However, for $d \geq 75 \AA$, the most stable phase is the single pancake configuration that concentrates the whole liquid on one of the walls, always at lower energy than the double-pancake configuration with the same total number of He atoms per unit length. A stable sandwich configuration at $d=50 \AA$ and a stable pancake configuration at $d=100$ $\AA$ have been drawn, as well as a metastable sandwich configuration at $d=100 \AA$ and a metastable two-pancakes configuration at $d=80 \AA$. The metastable systems are present on each side of the crossing. It becomes clear that for nanoscopic helium systems, capillary condensation between walls is possible -and thermodynamically stable- only up to a limiting separation. This feature should be taken into account when discussing the filling of a narrow wedge, since the approximation of the latter as two parallel plates applies only locally, i.e., the separation between walls increases with the distance from the apex.

To conclude, we believe that studies of wedge filling and wedge wetting by means of FRDFT may provide a powerful instrument to investigate the growth of nanoscopic helium systems under confinement by external potentials.

\section{ACKNOWLEDGMENTS}

F.A. acknowledges support from CESCA-CEPBA (HPC program).

\section{REFERENCES}

1. A. Hanke, M. Krech, F. Schlesener and S. Dietrich, Phys. Rev. E 60, 5163 (1999).

2. A. Checco, O. Gang and B. M. Ocko, Phys. Rev. Lett. 96, 056104 (2006).

3. P. Lenz and R. Lipowsky, Phys. Rev. Lett. 80, 1920 (1998)

4. E. Cheng and M. W. Cole, Phys. Rev. B 41, 9650 (1990).

5. M. Napiórkowski, W. Koch and S. Dietrich, Phys. Rev. B 45, 5760 (1992).

6. E. H. Hauge, Phys. Rev. A 46, 4994 (1992).

7. K. Rejmer, S. Dietrich and M. Napiórkowski Phys. Rev. E 60, 4027 (1999).

8. L. Bruschi, A. Carlin and G. Mistura, Phys. Rev. Lett. 89, 166101 (2002).

9. C. Rascón and A. O. Parry, Nature 407, 986 (2000).

10. E. S. Hernández et al., Phys. Rev. B 73, 245406 (2006).

11. F. Dalfovo, A. Lastri, L. Pricaupenko, S. Stringari and J. Treiner, Phys. Rev. B 52, 1193 (1995).

12. J. Klier, P. Leiderer. D. Reinelt and A. F. G. Wyatt, Phys. Rev. B 72, 245410 (2005).

13. A. Chizmeshya, M. W. Cole and E. Zaremba, J. Low Temp. Phys. 110, 677 (1998).

14. M. M. Calbi et al., J. Chem. Phys. 115, 9975 (2001). 